\title{
O efeito da floresta alagada na alimentação de três espécies de peixes onívoros em lagos de várzea da Amazônia Central, Brasil.
}

\author{
Luiz CLARO-JR ${ }^{1}$, Efrem FERREIRA², Jansen ZUANON², Carlos ARAUJO-LIMA ${ }^{2 \dagger}$
}

\begin{abstract}
RESUMO
As enchentes anuais dos rios na Amazônia alagam extensas áreas de floresta conhecidas como várzeas ou igapós. Estas áreas têm papel importante na vida dos peixes da região, pois são fontes de alimento e de abrigo. Acreditamos que o desmatamento destas áreas ocasiona prejuízos à ictiofauna principalmente pela diminuição da quantidade e diversidade de alimento disponível. O estudo da relação entre a quantidade de floresta e a dieta de Parauchenipterus galeatus (Auchenipteridae, Siluriformes), Mylossoma duriventre (Characidae, Characiformes) e Triportheus elongatus (Characidae, Characiformes) permitiu registrar pela primeira vez a influência direta da floresta alagada na ecologia alimentar de peixes na Amazônia Central.
\end{abstract}

\section{PALAVRAS-CHAVE}

Amazônia, peixes, alimentação, floresta alagada, lagos, desmatamento.

\section{Effects of flooded forest in the diet of three fish species in floodplain lakes of the Central Amazonia, Brazil.}

\begin{abstract}
The annual flooding of the rivers in the Amazonia extends over large forest areas known as várzeas (floodplain) or igapós (flooded forest). These areas play an important role in the life of the fishes, as a source of food and shelter. We believe that the deforestation of these areas may affect negatively the fish fauna mainly by decreasing the amount of food resources and consequently the diversity of food types available. The study of the relationship between the riparian forest cover and the diet of Parauchenipterus galeatus (Auchenipteridae, Siluriformes), Mylossoma duriventre (Characidae, Characiformes) and Triportheus elongatus (Characidae, Characiformes) allowed to refer for the first time to the direct influence of the flooded forest in the feeding ecology of fishes in the Central Amazonia.
\end{abstract}

\section{KEY WORDS}

Amazonia, fishes, feeding, flooded forest, lakes, deforestation.

Os peixes da Bacia Amazônica são submetidos a drásticas variações sazonais nas condições dos ambientes aquáticos, que implicam na necessidade de otimizar os ganhos energéticos na época mais favorável, a cheia (Goulding, 1980). Neste período ocorre um transbordamento do canal dos rios de águas brancas que invadem grandes áreas adjacentes (chamadas de várzea) e permitem que muitos organismos ocupem esses locais em busca de alimento e abrigo. É nesta época, também, que ocorre a frutificação de grande número de espécies de árvores da várzea, que com a ajuda da água, podem ter suas sementes dispersas para outros locais (Kubitzki \& Ziburski, 1994). Esses frutos e sementes são fontes energéticas importantes para a alimentação de peixes (Goulding, 1980; Waldhoff et al., 1996), incluindo espécies de grande valor comercial, como o tambaqui Colossoma macropomum (Araujo-Lima \& Goulding, 1998) (Figura 1).

No entanto, o impacto das ações antrópicas nas áreas de várzea vem aumentando ao longo dos tempos (Monteiro \& Sawyer, 2001). A ocupação desses locais tem sido feita com a derrubada da mata, tanto para a exploração de madeira como para agricultura, pois os solos da várzea são os solos amazônicos mais ricos em nutrientes (Ayres, 1993; Goulding et al., 1996). Em alguns locais da várzea do sistema Solimões-Amazonas existe também uma grande quantidade de gado bovino e bubalino (Goulding et al., 1996), espécies

${ }^{1}$ Programa de Pós-Graduação em Biologia de Água Doce e Pesca Interior (INPA) clarojr@inpa.gov.br

${ }^{2}$ Instituto Nacional de Pesquisas da Amazônia- INPA/CPBA, C.P. 478, 69011-970, Manaus, AM

${ }^{\dagger}$ In memoriam 


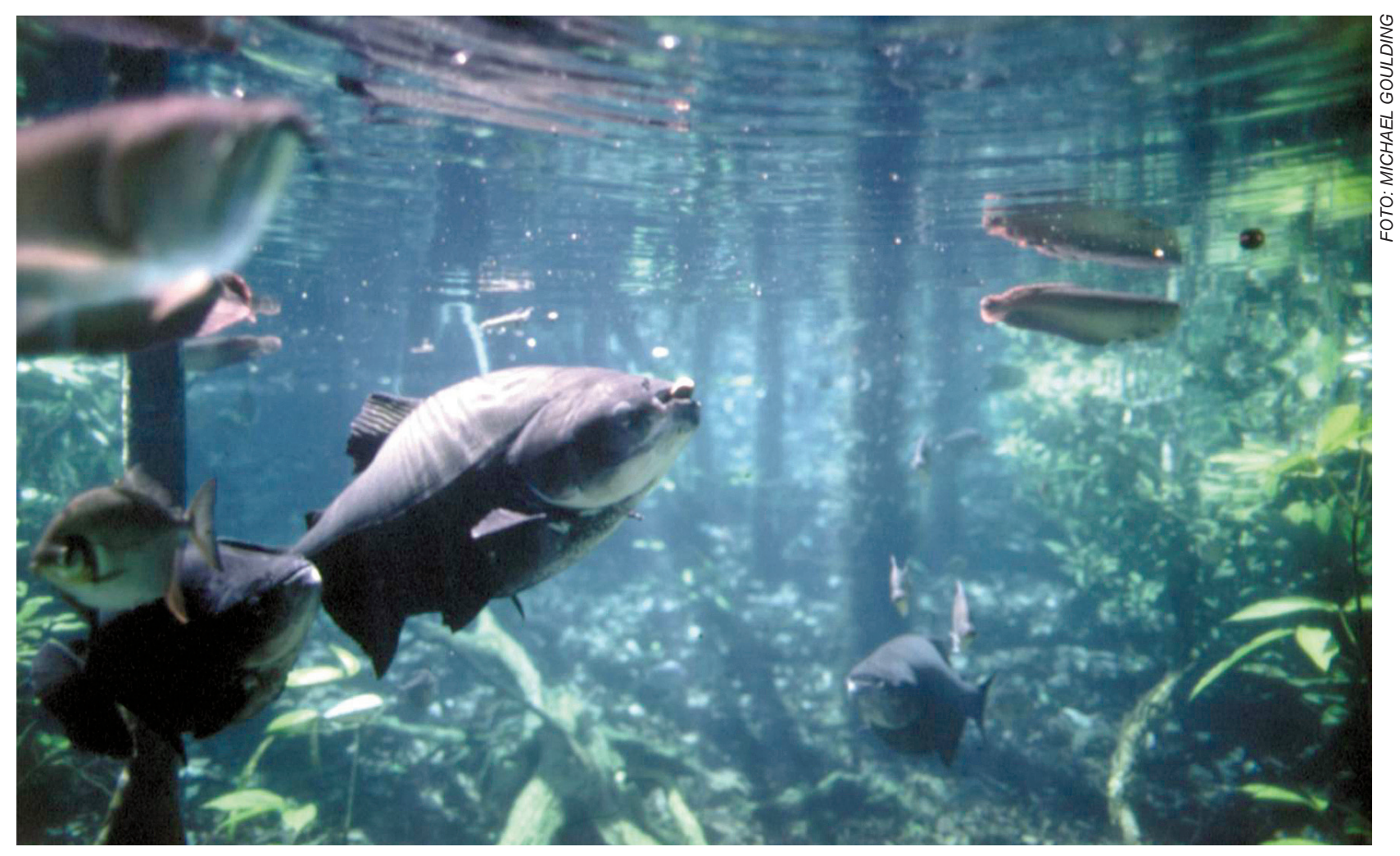

Figura 1 - Tambaqui (Colossoma macropomum) se alimentando no igapó.

exóticas que se alimentam de capins da várzea, principalmente o capim-membeca (Paspalum repens, Poaceae) que é um importante berçário para peixes (Sanchez-Botero \& Araujo-Lima, 2001) e habitat para muitos invertebrados (Junk, 1973). A substituição da floresta por pastos deve ocasionar mudanças nos hábitos alimentares dos peixes, pela redução na oferta de frutos, sementes e outras formas de matéria orgânica originada da floresta. Mesmo a substituição da cobertura florestal original por vegetação secundária (capoeiras) pode ter impactos negativos diretos sobre a ictiofauna. Em um experimento de cultivo, Roubach \& Saint-Paul, (1994) mostraram que a substituição, na composição de rações experimentais, de sementes de seringa (Hevea spp., Euphorbiaceae, árvore comum em florestas primárias de várzea), por sementes de embaúba (Cecropia sp. Moraceae, muito abundante em capoeiras), provocou impactos negativos na taxa de crescimento de tambaquis jovens.

Além da relação óbvia entre a floresta alagada e a fauna de peixes frugívoros, outros grupos tróficos também dependem da oferta de alimentos nesse ambiente. Uma grande quantidade e diversidade de invertebrados ocupam as florestas de várzea (Adis, 1997) e são consumidos por numerosas espécies de peixes (Goulding, 1980; Junk et al.,1997). Entre essas está o cangati, Parauchenipterus galeatus (Linnaeus, 1766) (Auchenipteridae, Siluriformes), o pacú-manteiga, Mylossoma duriventre (Cuvier, 1818)
(Characidae, Characiformes) e a sardinha, Triportheus elongatus (Gunther, 1864) (Characidae, Characiformes). Estes peixes apresentam porte médio e são abundantes em áreas de várzea, com ampla distribuição na Bacia Amazônica (Ferreira et al., 1998). O objetivo do presente trabalho foi avaliar a influência da área de floresta alagada na alimentação destas três espécies de peixes em lagos de várzea da Amazônia Central.

A escolha das espécies para análise baseou-se em três categorias básicas: estar presente em um número suficiente de lagos; apresentar um número razoável de exemplares coletados, para permitir uma análise estatística adequada e se alimentar de itens alóctones e autóctones Parauchenipterus galeatus, Mylossoma duriventre, e Triportheus elongatus foram as espécies que se enquadraram nestes pré-requisitos. Foram analisados 49 exemplares de $P$. galeatus capturados em oito lagos; 22 exemplares de $M$. duriventre e 48 exemplares de $T$. elongatus, capturados em sete lagos de várzea na Amazônia Central. Foi utilizada uma bateria de malhadeiras com malhas variando entre 30 e $160 \mathrm{~mm}$ e área total de $975 \mathrm{~m}^{2}$, colocadas dentro da floresta alagada. As redes foram armadas durante um período de 24 horas em cada lago, em junho de 2000. Os peixes capturados foram separados por espécie e fixados em formol 10\%. Após a biometria (comprimento padrão e peso) os estômagos foram retirados e preservados em álcool $70 \%$. Nas análises das dietas das espécies foram 
usados os métodos de freqüência de ocorrência e do volume relativo (Hyslop, 1980) e os dados apresentados na forma de Índice de Preponderância (IP) (Natarajan \& Jhingran, 1961), calculado pela fórmula:

$$
\mathrm{IP}=\left(\% \mathrm{O}_{\mathrm{i}} \cdot \% \mathrm{~V}_{\mathrm{i}}\right) / \Sigma^{\mathrm{i}=\mathrm{n}}\left(\% \mathrm{O}_{\mathrm{i}} \cdot \% \mathrm{~V}_{\mathrm{i}}\right)
$$

onde: $\mathrm{O}_{\mathrm{i}}$ é a freqüência de ocorrência do item $i$; e $\mathrm{V}_{\mathrm{i}} \mathrm{O}$ Volume relativo do item $i$.

A quantidade de floresta alagada nos lagos de várzea foi calculada a partir de imagens de radar (NASDA, 1996), após classificação da cobertura vegetal. Foi utilizado um raio de 4 $\mathrm{km}$ a partir do ponto de pesca para a definição da área de cálculo da cobertura florestal, que é a área potencial de ação dos equipamentos de pesca. Este raio representa a distância média que um dado peixe poderia percorrer em um dia, considerando um período de natação de 6 horas e velocidade de $18 \mathrm{~cm} / \mathrm{s}$. Para detalhes metodológicos vide Claro-Jr (2003).

Para verificar a relação entre a quantidade de floresta alagada e a participação de alimento de origem alóctone na dieta das espécies, foram utilizadas análises de regressão linear simples. Os dados das espécies $P$. galeatus e $T$. elongatus sofreram transformação $\ln (x+1)$ para quantidade de floresta alagada e arcosenoe $\sqrt{ } \mathbf{x}$, para os dados em porcentagem do IP e volume de alimento (Zar, 1999). Lagos que apresentaram somente um indivíduo coletado de uma dada espécie foram retirados das análises.

A dieta de $P$. galeatus consistiu principalmente de frutos, sementes e invertebrados terrestres (Tabela 1). Os principais invertebrados terrestres presentes no conteúdo estomacal foram formigas (Hymenoptera), cupins (Isoptera), grilos (Orthoptera) e aranhas (Araneae). O material autóctone consumido por $P$. galeatus era composto por insetos aquáticos (Odonata, Hemiptera e Ephemeroptera), outros invertebrados (Crustacea (Decapoda e Ostracoda) e Molusca); e peixes. Macrófitas aquáticas foram registradas no conteúdo estomacal, mas sua baixa ocorrência, indica que podem ter sido ingeridas acidentalmente junto com outras presas. A relação entre a quantidade de itens alóctones na dieta de $P$. galeatus e a quantidade de floresta, foi significativamente positiva $\left(\mathrm{Y}=-2,5+11,09 \mathrm{x} ; \mathrm{r}^{2}=0,47 ; \mathrm{p}=0,05\right)$ (Figura 2a).

A dieta de $M$. duriventre consistiu principalmente de itens alóctones como frutos, sementes e invertebrados terrestres
(Tabela 2). O material autóctone consumido por $M$. duriventre foi composto de zooplâncton, insetos aquáticos, macrófitas e detrito. A relação entre a quantidade de invertebrados alóctones na dieta de $M$. duriventre e a quantidade de floresta, foi significativamente positiva $\left(\mathrm{Y}=-23,3+0,03 \mathrm{x} ; \mathrm{r}^{2}=0,84 ; \mathrm{p}<0,01\right)$ (Figura 2b). Os principais invertebrados terrestres presentes na dieta de $M$. duriventre foram insetos (Hymenoptera; Formicidae e Coleoptera) e aranhas (Araneae).

A dieta de T. elongatus também consistiu principalmente de frutos, sementes e invertebrados terrestres (Tabela 3). Os principais invertebrados terrestres presentes na dieta de $T$. elongatus foram insetos (Isoptera, Orthoptera, Homoptera e Hymenoptera (Formicidae)) e aranhas (Araneae). O material autóctone consumido por T. elongatus foi composto por insetos aquáticos (Hemiptera, Ephemeroptera, Odonata). A relação entre a quantidade de itens alóctones na dieta de $T$. elongatus e a quantidade de floresta foi significativamente positiva ( $\left.\mathrm{Y}=-102,3+25,9 \mathrm{x} ; \mathrm{r}^{2}=0,59 ; \mathrm{p}<0,05\right)$ (Figura2c). Os alimentos de origem vegetal que esta espécie consumiu foram sementes de Cecropia sp. (Moraceae) e de Pseudobombax munguba (Bombacaceae) e flores.

Estas espécies de peixes utilizam os recursos de origem alóctone como fonte principal de energia e suas dietas parecem refletir diretamente mudanças na quantidade de floresta alagada. Como foi o caso de $P$. galeatus, que em lagos com maior quantidade de floresta somente consumiu frutos, sementes e invertebrados terrestres. Já nos lagos que apresentaram menor quantidade de floresta encontrou-se peixes e decápodes no conteúdo estomacal desta espécie. Na Bacia do rio Paraná, $P$. galeatus apresentou mudança no hábito alimentar em função da disponibilidade de alimento (verão - inverno), tanto em uma área de reservatório artificial como em rios de cabeceira (Andrian \& Barbieri, 1996).

Sementes de Cecropia sp. (embaúba), comuns em florestas secundárias, foram uma das mais ingeridas por $P$. galeatus em lagos com pouca área de floresta inundada, enquanto que, nos lagos com maior quantidade de floresta, foram ingeridas sementes maiores, como Vitex sp. (Verbenaceae), Ilex sp. (Aquifoliaceae) e Macrolobium sp. (Fabaceae), típicas de floresta de várzea. Tal resultado indica que esta espécie de peixe também mudou o tipo de semente ingerida nos lagos mais impactados com o desmatamento,
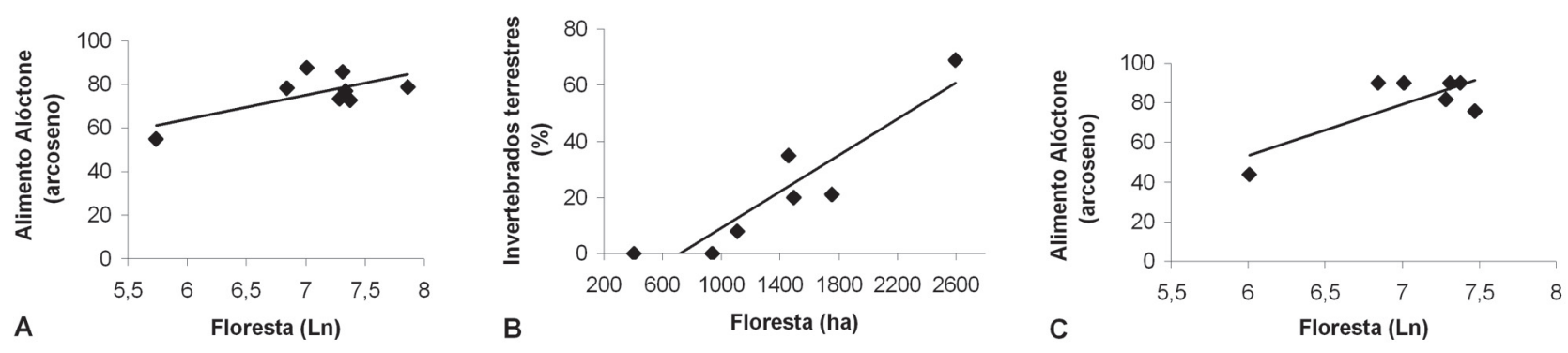

Figura 2 - Relação entre a quantidade de floresta alagada e o Índice de Preponderância de itens alóctones na dieta de $P$. galeatus (A); e o volume de invertebrados terrestres na dieta de $M$. duriventre (B); e o volume itens alóctones na dieta de T. elongatus (C). 


\section{ACTA \\ AMAZONICA}

O EFEITO DA FLORESTA ALAGADA NA ALIMENTAÇÃO DE TRÊS ESPÉCIES

DE PEIXES ONÍVOROS EM LAGOS DE VÁRZEA DA AMAZÔNIA CENTRAL, BRASIL. provavelmente refletindo diferenças na disponibilidade desses recursos alimentares.

No processo de sucessão de uma floresta primária que foi derrubada, é comum que a mata secundária passe a ser inicialmente dominada por Cecropia sp., uma planta pioneira cujos frutos são utilizados pelos peixes como alimento. Porém, o valor nutricional de seus frutos é baixo quando comparado com os de frutos e sementes de plantas de mata primária, como Hevea brasiliensis (seringa) e Astrocaryum jauari (jauari) (Waldorf et al., 1996). Assim, com o desmatamento das várzeas os peixes estão sendo prejudicados, tanto em relação à disponibilidade de frutos e sementes como pelo baixo valor nutricional das novas fontes de alimento. Isso pode comprometer o crescimento dos peixes, como visto por

Tabela 1 - Quantidade de floresta alagada (ha), número de exemplares e Índice de Preponderância (IP) de cada tipo de alimento (\%) na dieta de $P$ galeatus em oito lagos de várzea da Amazônia Central.

\begin{tabular}{|c|c|c|c|c|c|c|c|c|c|c|}
\hline \multirow[b]{2}{*}{ Lagos } & \multirow{2}{*}{$\begin{array}{l}\text { Floresta } \\
\text { (ha) }\end{array}$} & \multirow{2}{*}{$\begin{array}{c}\text { Número } \\
\text { (n) }\end{array}$} & \multicolumn{3}{|c|}{ Alóctone } & \multicolumn{5}{|c|}{ Autóctone } \\
\hline & & & $\begin{array}{l}\text { Frutos e } \\
\text { sementes }\end{array}$ & $\begin{array}{c}\text { Invertebrado } \\
\text { terrestre }\end{array}$ & Subtotal & $\begin{array}{l}\text { Invertebrado } \\
\text { aquático }\end{array}$ & $\begin{array}{c}\text { Outros } \\
\text { invertebrados }\end{array}$ & Peixe & Macrófita & Subtotal \\
\hline MA-25 & 311 & 2 & 53,2 & 14,0 & 67,2 & 0,0 & 32,8 & 0,0 & 0,0 & 32,8 \\
\hline$M A-10$ & 937 & 14 & 89,1 & 6,8 & 95,9 & 0,7 & 3,1 & 0,0 & 0,3 & 4,1 \\
\hline MA-15 & 1108 & 7 & 88,0 & 11,9 & 99,8 & 0,0 & 0,1 & 0,0 & 0,0 & 0,2 \\
\hline MA-08 & 1458 & 3 & 78,8 & 13,1 & 91,9 & 3,6 & 4,5 & 0,0 & 0,0 & 8,1 \\
\hline MA-23 & 1495 & 2 & 98,2 & 1,3 & 99,5 & 0,5 & 0,0 & 0,0 & 0,0 & 0,5 \\
\hline MA-24 & 1533 & 1 & 0,0 & 95,0 & 95,0 & 5,0 & 0,0 & 0,0 & 0,0 & 5,0 \\
\hline MA-22 & 1596 & 14 & 88,5 & 2,8 & 91,3 & 6,0 & 2,7 & 0,0 & 0,0 & 8,7 \\
\hline MA-04 & 2593 & 5 & 68,5 & 27,9 & 96,4 & 3,6 & 0,0 & 0,0 & 0,0 & 3,6 \\
\hline
\end{tabular}

Tabela 2 - Quantidade de floresta alagada (ha), número de exemplares e Índice de Preponderância (IP) de cada tipo de alimento (\%) na dieta de $M$. duriventre em sete lagos de várzea da Amazônia Central.

\begin{tabular}{lccccccc}
\hline \hline Lagos & $\begin{array}{c}\text { Floresta } \\
\text { (ha) }\end{array}$ & Número $(\mathrm{n})$ & Detrito & Macrófita & $\begin{array}{c}\text { Invertebrado } \\
\text { aquático }\end{array}$ & $\begin{array}{c}\text { Invertebrado } \\
\text { terrestre }\end{array}$ & $\begin{array}{c}\text { Semente/ } \\
\text { Fruto/Flor }\end{array}$ \\
\hline MA-11 & 407 & 2 & 0,0 & 0,0 & 20,0 & 0,0 & 80,0 \\
MA-10 & 937 & 2 & 0,0 & 0,0 & 0,0 & 0,0 & 100,0 \\
MA-15 & 1108 & 6 & 0,0 & 0,0 & 0,0 & 3,8 & 96,2 \\
MA-08 & 1458 & 3 & 0,0 & 0,0 & 0,0 & 35,0 & 65,0 \\
MA-23 & 1495 & 2 & 3,7 & 29,6 & 0,0 & 14,8 & 51,9 \\
MA-17 & 1753 & 5 & 0,0 & 0,0 & 0,0 & 13,4 & 86,6 \\
MA-04 & 2593 & 2 & 0,0 & 0,0 & 0,9 & 53,3 & 45,8 \\
\hline Total & & 22 & & & & & \\
\hline \hline
\end{tabular}

Tabela 3 - Quantidade de floresta alagada (ha), número de exemplares e Índice de Preponderância (IP) de cada tipo de alimento (\%) na dieta de T. elongatus em sete lagos de várzea da Amazônia Central.

\begin{tabular}{|c|c|c|c|c|c|}
\hline Lagos & Floresta (ha) & Número (n) & Invertebrado aquático & Invertebrado terrestre & Semente/ Fruto/Flor \\
\hline$\overline{M A-11}$ & 407 & 2 & 53,6 & 43,2 & 3,2 \\
\hline$M A-10$ & 937 & 6 & 0,0 & 5,2 & 94,7 \\
\hline MA-15 & 1108 & 3 & 0,0 & 3,1 & 96,9 \\
\hline MA-08 & 1458 & 17 & 0,3 & 32,0 & 67,7 \\
\hline MA-23 & 1495 & 3 & 0,0 & 35,0 & 65,0 \\
\hline$M A-22$ & 1596 & 15 & 0,0 & 1,6 & 98,4 \\
\hline MA-17 & 1753 & 2 & 6,0 & 54,0 & 40,0 \\
\hline Total & & 48 & & & \\
\hline
\end{tabular}




\section{ACTA \\ AMAZONICA}

O EFEITO DA FLORESTA ALAGADA NA ALIMENTAÇÃO DE TRÊS ESPÉCIES

DE PEIXES ONIVIOROS EM LAGOS DE VÁRZEA DA AMAZÔNIA CENTRAL, BRASIL.
Roubach \& Saint-Paul, (1994), em experimentos de piscicultura, e conseqüentemente influenciar negativamente no sucesso da reprodução e na taxa de recrutamento.

Claro-Jr (2003) mostrou que quanto maior a quantidade de floresta alagada, maior a biomassa de peixes que consomem itens alóctones em lagos de várzea da Amazônia Central; porém a abundância e a riqueza dessas espécies não tiveram relações significativas com a cobertura florestal. Isso indica que a maior quantidade de floresta resulta em maior quantidade de peixes, mas com algumas espécies aumentando sua dominância nas comunidades.

Muitas pesquisas têm sido realizadas para se entender os efeitos da fragmentação florestal nos ecossistemas terrestres amazônicos (e.g., Gascon \& Moutinho, 1998). Até onde pudemos verificar, este é o primeiro estudo que relaciona diretamente a dieta de espécies de peixes à quantidade de floresta ripária disponível, para habitats de várzea.

Este é o início de investigações que estão sendo feitas para tentar avaliar os ganhos e perdas econômicas resultantes do desmatamento da várzea. Estudos enfocando o impacto antrópico na várzea ainda são recentes, mas faz-se necessário entender as conseqüências do crescimento populacional humano na Amazônia e tentar minimizar as perdas potenciais, tanto na pesca como na qualidade de vida das populações humanas.

\section{AGRADECIMENTOS}

Ao Instituto Nacional de Pesquisas da Amazônia através do PPI- 3020 e ao projeto cooperação Brasil/Espanha. Ao CNPq com o suporte da bolsa de mestrado.

\section{BIBLIOGRAFIA CITADA}

Adis, J. 1997. Terrestrial invertebrates: survival strategies, group spectrum, dominance and activity patterns. In: Junk, W.J. (Ed). The Central Amazon Floodplain: Ecology of a pulsing system. Springer-Verlag. Berlin, Germany. p.299-318.

Andrian, I.F.; Barbieri, G. 1996. Espectro alimentar e variação sazonal e espacial na composição da dieta de Parauchenipterus galeatus Linnaeus, 1766, (Siluriformes, Auchenipteridae) na região do reservatório de Itaipu, PR. Rev. Brasil. Biol., 56(2):409-422

Araujo-Lima, C.A.R.M.; Goulding, M. 1998. Os frutos do Tambaqui: ecologia, conservação e cultivo na Amazônia. Sociedade Civil Mamirauá/ CNPq/ Rainforest Alliance. Brasília, DF. 186pp.

Ayres, J.M. 1993. As matas de várzea do Mamirauá. Sociedade Civil Mamirauá/ CNPq/ Rainforest Alliance. Brasília, DF. 123pp.

Claro-Jr, L.H. 2003. A influência da floresta alagada na estrutura trófica de comunidades de peixes em lagos de várzea da Amazônia Central. Dissertação de Mestrado, INPA/UFAM. Manaus, Amazonas. 61pp.

Ferreira, E.J.G.; Zuanon, J.A.S.; Santos, G.M. 1998. Peixes Comerciais do Médio Amazonas: região de Santarém, Pará. Edições IBAMA. Brasília, DF. 214pp.
Gascon, C.; Moutinho, P. 1998. Floresta Amazônica: Dinâmica Regeneração e Manejo. MCT/INPA, Manaus, Brasil. 373pp.

Goulding, M. 1980. The fishes and the forest. Explorations in Amazonian Natural History. University of California Press. Berkeley, USA. 280pp.

Goulding, M.; Smith, N.J.H.; Mahar, D.J. 1996. Floods of fortune: ecology and economy along the Amazon. Columbia University Press, New York, USA. 193pp

Hyslop, E.J. 1980. Stomach contents analysis - a review of methods and their application. J. Fish Biol., 17:411-429

Junk, W.J. 1973. Investigations on the ecology and productionbiology of the "floating meadows" (Paspalo-Echinocloetum) on the middle Amazon. Part II: The aquatic fauna in the root zone of floating vegetation. Amazoniana, 4(1):9-12.

Junk, W.J.; Soares, M.G.M.; Saint-Paul, U. 1997. The Fish. In: Junk, W.J. (Ed.) The Central Amazon Floodplain: Ecology of a pulsing system. Springer-Verlag. Berlin, Germany. p.385-408.

Monteiro, M.P.; Sawyer, D. 2001. Diagnóstico demográfico, socioeconômico e de pressão antrópica na região da Amazônia Legal. In: Capobianco, J.P.R.; Veríssimo, A.; Moreira, A.; Sawyer, D.; Santos, I.; Pinto (Eds). Biodiversidade na Amazônia Brasileira: avaliação e ações prioritárias para a conservação, uso sustentável e repartição de benefícios. São Paulo: Estação Liberdade/ Instituto. p.308-320.

NASDA. 1996. Imagem de radar.

Natarajan, A.V.; Jhingran, A.G. 1961. Index of Preponderance - a method of grading the food elements in the stomach analysis of fishes. Indian J. Fish, 8:54-59

Kubitzki, K.; Ziburski, A. 1994. Seed dispersal in flood plain forest of Amazonia. Biotropica, 26(1):30-43

Roubach, R.; Saint-Paul, U. 1994. Use of fruits and seeds from Amazonian inundated forest in feeding trial with Colossoma macropomum (Cuvier, 1818) (Pisces, Characidae). J. Appl. Ichthyol., 10:134-140

Sanchez-Botero, J.I.S.; Araujo-Lima, C.A.R.M. 2001. As macrófitas aquáticas como berçário para a ictiofauna da várzea do rio Amazonas. Acta Amazonica, 31(3):437-448

Waldhoff, D.; Sant-Paul, U.; Furch. B. 1996. Value of fruits and seeds from the floodplain forests of central Amazonia as food resource for fish. Ecotropica, 2:143-156

Zar, J.H. 1999. Biostatistical analysis. $4^{\mathrm{a}}$ edição. Prentice-Hall, Inc., Englewood cliffs. N.J., USA. 663pp.

RECEBIDO EM 11/06/2003

ACEITO EM 19/11/2003 
